\title{
Albumin-to-alkaline phosphatase ratio at diagnosis predicts survival in patients with metastatic non-small-cell lung cancer
}

This article was published in the following Dove Press journal:

OncoTargets and Therapy

\section{Dan Li* \\ $\mathrm{He} \mathrm{Yu*}$ \\ Weimin Li \\ Department of Respiratory and Critical Care Medicine, West China Hospital, Sichuan University, Chengdu, People's Republic of China \\ *These authors contributed equally to this work}

Correspondence: Weimin $\mathrm{Li}$

Department of Respiratory and Critical

Care Medicine, West China Hospital,

Sichuan University, No. 37, Guoxue Alley,

Chengdu 61004I, Sichuan Province,

People's Republic of China

Tel +86 2885582944

Fax +862885582944

Email weimi003@I63.com
Background: Previous studies have reported that the albumin-to-alkaline phosphatase ratio (AAPR), a novel blood biomarker-based index, is associated with clinical outcome in several cancers. However, data relating to lung cancer are rare. This study was performed to clarify the clinical significance of AAPR in patients with metastatic non-small-cell lung cancer (NSCLC). Methods: In total, 290 stage IV NSCLC patients were enrolled in this retrospective study. Associations between serum enzyme levels and clinical characteristics were analyzed using the Mann-Whitney U-test or chi-squared test. Kaplan-Meier survival analysis and Cox's proportional hazard regression model were adopted to assess the prognostic value of AAPR for overall survival (OS).

Results: The optimal cut-off points for AAPR and lactate dehydrogenase (LDH) were 0.36 and 265.5 U/L, respectively. Patients with AAPR $\leq 0.36$ had apparently longer survival than those with AAPR $>0.36$ (13 vs 7 months, $P<0.001)$. Furthermore, AAPR was an independent predictor of $\mathrm{OS}$ in metastatic NSCLC in multivariate analysis (HR=0.657, 95\% $\mathrm{CI}=0.504-0.856, P<0.01)$. The prognostic impact of $\mathrm{LDH}$ for survival of NSCLC populations was also validated in this study $(\mathrm{HR}=1.462,95 \% \mathrm{CI}=1.070-1.999, P<0.05)$.

Conclusion: Elevated AAPR can be an independent favorable prognostic indicator in metastatic NSCLC.

Keywords: AAPR, LDH, NSCLC, prognosis

\section{Introduction}

Lung cancer remains one of the most prevalent malignancies and the leading cause of cancer-related death worldwide. ${ }^{1}$ Approximately $85 \%$ of primary lung cancers are non-small cell lung cancer (NSCLC), and more than half are metastatic NSCLC at the time of initial diagnosis, owing to a lack of typical symptoms in early disease. $^{2}$ Patients with metastatic NSCLC can be treated with chemotherapy, radiotherapy, or both. Thanks to advances in treatment, they can benefit from targeted therapy as well. ${ }^{3}$ However, the prognosis for metastatic NSCLC is extremely poor, with a 5-year survival rate of $3.9 \% .^{2}$ Although the TNM classification system has the most marked discriminatory capacity for survival of cancers, it has reached its limits in metastatic NSCLC patients. Therefore, finding novel and efficient biomarkers to help predict clinical outcomes and guide clinical decision-making for metastatic NSCLC is of great importance.

Carcinogenesis and tumor progression always lead to abnormal serum enzyme synthesis, sometimes even before disease is clinically detectable. ${ }^{4}$ These enzymes 
can serve as useful indicators for tumor detection and monitoring. Alkaline phosphatase (ALP) is a hydrolase enzyme which is responsible for removing phosphate groups. Elevated serum ALP levels can be seen in liver, kidney, and bone diseases. ${ }^{5}$ ALP has been found to be associated with advanced cancer status. ${ }^{6,7}$ Lactate dehydrogenase (LDH) is another serum enzyme, which mainly participates in the conversion of pyruvate to lactate and is related to tumor metabolism. Numerous studies have found that $\mathrm{LDH}$ levels are elevated in various types of cancer, including NSCLC. ${ }^{8-11}$

Albumin (ALB), one of the most commonly used methods to assess nutritional status, usually shows no or only slight changes in the early stages of cancer. But as the disease progresses, malnutrition and inflammation lead to ALB synthesis being suppressed and degradation increasing, which result in ALB levels dropping significantly. ${ }^{12}$ The decreased ALB in cancer patients can result in shorter survival and increased cancer-related mortality. ${ }^{13,14}$ Besides ALB alone, several ALB-based markers, such as the Glasgow Prognostic Score, ${ }^{15,16}$ Advanced Lung Cancer Inflammation Index, ${ }^{17}$ and Prognostic Nutritional Index, ${ }^{18,19}$ have also been shown to correlate with survival in various cancers.

In the present study, we merge the parameters ALB and ALP to form a novel index called the albumin-to-alkaline phosphatase ratio (AAPR). The AAPR has been revealed as a prognostic indicator for hepatocellular carcinoma ${ }^{20}$ and nasopharyngeal carcinoma. ${ }^{21}$ However, whether AAPR can predict survival in NSCLC patients has not yet been investigated. Thus, we performed this singlecenter retrospective study to explore for the first time the prognostic impact of AAPR and its relationship with clinical characteristics in patients with metastatic NSCLC. The correlation between $\mathrm{LDH}$ and prognosis of metastatic NSCLC was also demonstrated.

\section{Materials and methods}

\section{Participants}

We retrospectively reviewed newly diagnosed stage IV NSCLC patients in West China Hospital from March 2007 to December 2013. The inclusion criteria included the following: 1) pathologically confirmed NSCLC; 2) clinically diagnosed metastasis; 3) serum ALB and ALP measured at diagnosis; and 4) follow-up data were available. Patients with a history of a second primary malignancy, detectable infections, and known liver or renal or bone diseases were excluded from the study. In total, 290 NSCLC patients who met the inclusion criteria were eventually enrolled in the study. The West China Hospital Research Ethics Committee approved the study and signed informed consent from patients was waived because of the retrospective nature of the analysis. All patient data were treated with confidentiality, in accordance with the Declaration of Helsinki.

\section{Clinical and follow-up data}

We collected baseline and clinical characteristics of patients from the hospital medical system, including age, gender, smoking history, histology type, metastatic characteristics, and medication. Patients who had smoked fewer than 100 cigarettes in their lifetime were defined as non-smokers. Histology type was identified based on the WHO criteria established in $2004 .^{22}$ Tumor stage was defined according to the seventh edition of the TNM classification. ${ }^{23}$ Furthermore, pretreatment laboratory data covering ALB, ALP, and LDH were extracted. The AAPR was calculated by dividing the serum ALB by the serum ALP level.

The primary outcome of the study was patients dead of lung cancer. Time from diagnosis to death was measured as overall survival (OS). We obtained survival information by interviewing patients on the telephone or by checking medical records. The last follow-up was in October 2017.

\section{Statistical analysis}

The optimal cut-off values for the AAPR and LDH levels were determined using a biostatistical tool called Cutoff Finder (http://molpath.charite.de/cutoff/). ${ }^{24}$ Statistical analyses were performed with SPSS software (version 22; IBM Corp., Armonk, NY, USA). Continuous variables were presented as medians and ranges, and later were transformed into dichotomous variables presented as frequencies and percentages. Comparison of clinicopathological characteristics between different groups was conducted by the Mann-Whitney U-test for continuous variables and chisquared test for dichotomous variables. Associations between prognostic factors and OS were assessed by the Kaplan-Meier method and tested by the log-rank test, while Kaplan-Meier survival curves were drafted in Prism 5 (version 5.01; GraphPad Software, La Jolla, CA, USA). For univariate and multivariate survival analyses, Cox's proportional hazard regression model was adopted. Candidate variables with a $P$-value less than 0.2 in univariate analysis were included in the multivariate model. A two-sided $P$-value less than 0.05 was considered statistically significant. 


\section{Results}

\section{Clinical characteristics of patients}

Overall, 290 patients were involved in the study and their clinical characteristics are summarized in Table 1. Among these, $170(60.7 \%)$ were males, 128 (44.1\%) were aged older than 60 years (mean: 55 years; range: $25-90$ years), and $139(47.9 \%)$ had a current/past history of smoking. According to the histology, 215 out of 290 patients (74.1\%) had an adenocarcinoma, while 60 patients (20.7\%) had a squamous cell carcinoma and 15 (5.2\%) had other types. At the time of diagnosis, the majority of the patients (242; 83.4\%) had developed two or fewer metastasis sites and nearly half of the patients $(140 ; 48.3 \%)$ had liver/bone metastasis. In addition, more than half of the patients (196; $67.6 \%$ ) had received some form of systemic therapy, whereas the remaining patients $(94 ; 32.4 \%)$ had not.

Using Cutoff Finder, an R-software engineered web-based system, we identified that the optimal cut-off values for AAPR and LDH were 0.36 and $265.5 \mathrm{U} / \mathrm{L}$, respectively (Figure 1). Thus, 201 patients $(69.3 \%)$ with AAPR $>0.36$ were categorized into the high AAPR group and the other 89 patients (30.7\%) with AAPR $\leq 0.36$ into the low AAPR group. When dividing the patients by level of LDH, 234 patients $(80.7 \%)$ had LDH $>265.5 \mathrm{U} / \mathrm{L}$ and $56(19.3 \%)$ had LDH $\leq 265.5 \mathrm{U} / \mathrm{L}$.

The distribution of clinical characteristics between the AAPR $\leq 0.36$ and AAPR $>0.36$ groups is depicted in Table 1 .

Table I Distribution of baseline characteristics of 290 metastatic NSCLC patients according to AAPR level

\begin{tabular}{|c|c|c|c|c|}
\hline Characteristics & Total, n (\%) & AAPR $\leq 0.36, \mathrm{n}(\%)$ & AAPR $>0.36, n(\%)$ & $P$-value \\
\hline Number of patients & 290 & $89(30.7)$ & $20 \mathrm{I}(69.3)$ & \\
\hline Age (years) & & & & 0.898 \\
\hline$\leq 60$ & $162(55.9)$ & $49(55.1)$ & $113(56.2)$ & \\
\hline$>60$ & $128(44.1)$ & $40(44.9)$ & $88(43.8)$ & \\
\hline Gender & & & & 0.696 \\
\hline Male & $176(60.7)$ & $56(62.9)$ & $120(59.7)$ & \\
\hline Female & II4 (39.3) & $33(37.1)$ & $81(40.3)$ & \\
\hline Smoking status & & & & 1.000 \\
\hline Current/past & $139(47.9)$ & $43(48.3)$ & $96(47.8)$ & \\
\hline Never & $151(52.1)$ & $46(51.7)$ & $105(52.2)$ & \\
\hline Histology & & & & 0.502 \\
\hline SCC & $60(20.7)$ & $15(16.9)$ & $45(22.4)$ & \\
\hline ADC & $215(74.1)$ & $70(78.7)$ & $145(72.1)$ & \\
\hline Others & $15(5.2)$ & $4(4.5)$ & II (5.5) & \\
\hline Number of metastasis sites & & & & $0.001 *$ \\
\hline$\leq 2$ & $242(83.4)$ & $64(71.9)$ & $178(88.6)$ & \\
\hline$>2$ & $48(16.6)$ & $25(28.1)$ & $23(11.4)$ & \\
\hline Liver/bone metastasis & & & & $0.011 *$ \\
\hline Positive & $140(48.3)$ & $53(37.9)$ & $87(62.1)$ & \\
\hline Negative & $150(51.7)$ & $36(24.0)$ & $114(76)$ & \\
\hline Systemic therapy & & & & $0.007^{*}$ \\
\hline Yes & $196(67.6)$ & $50(56.2)$ & $146(72.6)$ & \\
\hline No & $94(32.4)$ & $39(43.8)$ & $55(27.4)$ & \\
\hline Albumin $(g / L)$, median (range) & $37.97(23.5-64.8)$ & $35.32(23.5-45.9)$ & $39.14(28.2-64.8)$ & $0.000 *$ \\
\hline ALP (U/L), median (range) & $121.73(1.46-2,170.0)$ & $221.80(79.0-2,170.0)$ & $77.43(1.46-119.0)$ & $0.000 *$ \\
\hline $\mathrm{LDH}(\mathrm{U} / \mathrm{L})$ & & & & $0.00 I^{*}$ \\
\hline$\leq 265.5$ & $234(80.7)$ & $61(68.5)$ & $173(86.1)$ & \\
\hline$>265.5$ & $56(19.3)$ & $28(31.5)$ & $28(13.9)$ & \\
\hline
\end{tabular}

Note: $* P<0.05$.

Abbreviations: AAPR, albumin-to-alkaline phosphatase ratio; ADC, adenocarcinoma; ALP, alkaline phosphatase; LDH, lactate dehydrogenase; NSCLC, non-small-cell lung cancer; SCC, squamous cell carcinoma. 

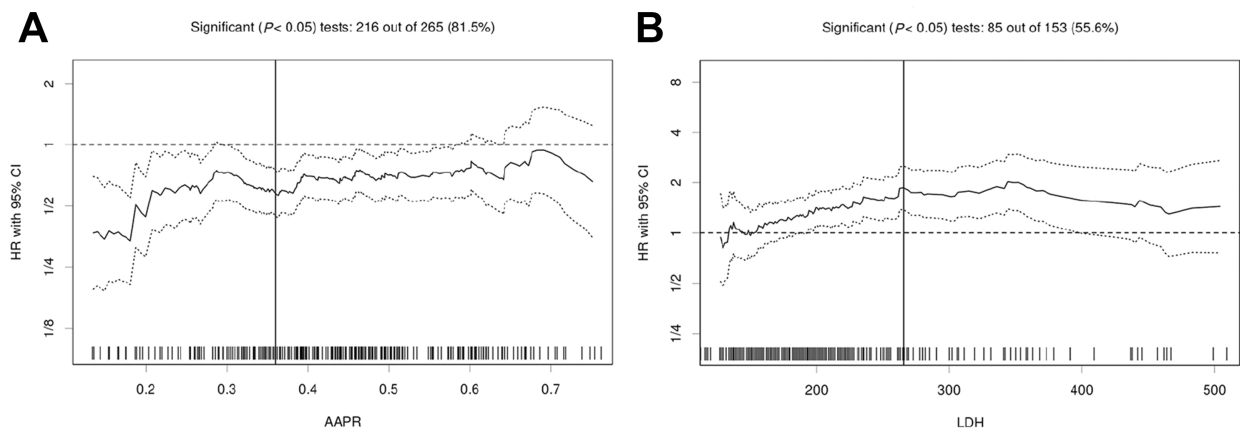

Figure I Hazard ratios for overall survival independent of cut-off point for (A) albumin-to-alkaline phosphatase ratio (AAPR) and (B) lactate dehydrogenase (LDH) in patients with metastatic non-small-cell lung cancer. The vertical line shows the optimal cut-off point with the most significant split (log-rank test). The plots were generated using Cutoff Finder.

Patients with elevated AAPR were more likely be those who harbored two or fewer metastasis sites, had undergone systemic therapy, had no liver/bone metastasis, and had LDH $\leq 265.5 \mathrm{U} / \mathrm{L}$ (all $P<0.05$ ). However, no significant differences were found regarding age, gender, smoking status, and tumor histology type between the two groups (all $P>0.05$ ).

A comparison of serum AAPR, ALP, LDH, and ALB levels in NSCLC patients with or without liver/bone metastasis is shown in Table 2. The results revealed that patients without liver/bone metastasis had higher AAPR and lower ALP than those with liver/bone metastasis $(0.47$ vs $0.40, P<0.001 ; 80.17$ vs $95.40 \mathrm{U} / \mathrm{L}, P<0.001$, respectively), while LDH and ALB levels in these two groups were not significantly different (both $P>0.05$ ).

\section{Kaplan-Meier survival analysis of AAPR for OS}

The median follow-up time of the 290 NSCLC patients was 16 months (range: 1-84 months). Altogether, 277 patients died during the follow-up period. A difference in prognosis was apparent between patients in the high AAPR group and patients in the low APPR group (13 months vs 7 months, $P<0.001)$ (Figure 2A). Later, stratified analyses were conducted. No significant difference was found in patients with current/past smoking divided by level of AAPR $(P>0.05)$ (Figure 2B), while in patients without smoking history, those with high AAPR showed significantly better survival than those with low AAPR $(P<0.001)$ (Figure $2 \mathrm{C})$. A similar survival trend was observed when patients were stratified into adenocarcinoma $(P<0.001)$ (Figure 2D), but not in nonadenocarcinoma $(P>0.05)$ (Figure 2E). Furthermore, we analyzed the relationship between AAPR and OS in patients with two or fewer metastasis sites and those with more than two, patients with and those without systemic therapy, and patients with and those without liver/bone metastasis, respectively. All of the results indicated that patients with elevated AAPR survived longer (all $P<0.05$ ) (Figure $\mathrm{S} 1$ ).

\section{Univariate and multivariate analyses for OS}

APPR level, LDH level, and systemic therapy were found to be significantly correlated with OS of metastatic NSCLC patients in univariate analysis (all $P<0.001$ ), while age, gender, smoking status, and tumor histology type were not (all $P>0.05$ ). In particular, the association between number of metastatic sites at diagnosis and OS was at the very edge of significance $(P=0.053)$. Four variables with a $P$-value less than 0.2 in univariate analysis were enrolled in multivariate analysis. The result revealed that AAPR was an independent

Table 2 Correlations between serum enzyme levels and liver/bone metastatic characteristics in NSCLC patients

\begin{tabular}{|l|l|l|l|}
\hline Characteristics & Liver/bone metastasis & P-value \\
\hline Median (range) & Positive & \multicolumn{1}{l|}{ Negative } \\
\hline AAPR & $0.40(0.02-0.74)$ & $0.47(0.02-44.38)$ & $0.000^{*}$ \\
ALP (U/L) & $95.40(47.00-1,573.00)$ & $80.17(1.46-2,170.00)$ & $0.000^{*}$ \\
LDH (U/L) & $201.50(117.00-966.00)$ & $189.00(46.00-691.00)$ & 0.078 \\
Albumin (g/L) & $38.40(26.60-51.30)$ & $38.06(23.50-64.80)$ & 0.641 \\
\hline
\end{tabular}

Note: $* P<0.05$.

Abbreviations: AAPR, albumin-to-alkaline phosphatase ratio; ALP, alkaline phosphatase; LDH, lactate dehydrogenase; NSCLC, non-small-cell lung cancer. 
A

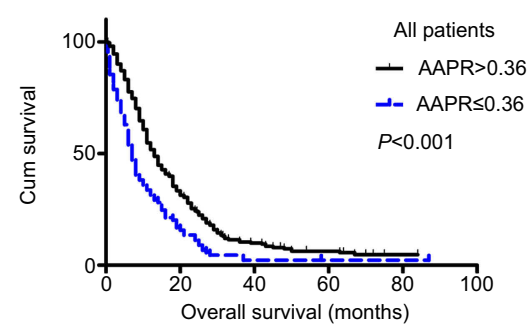

C

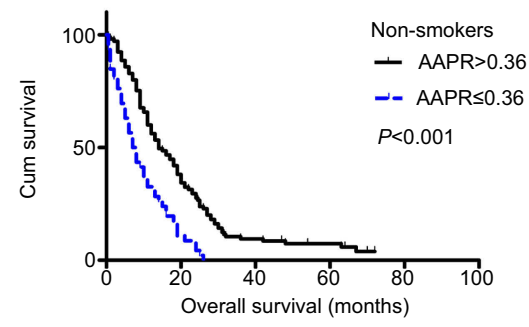

$\mathbf{E}$

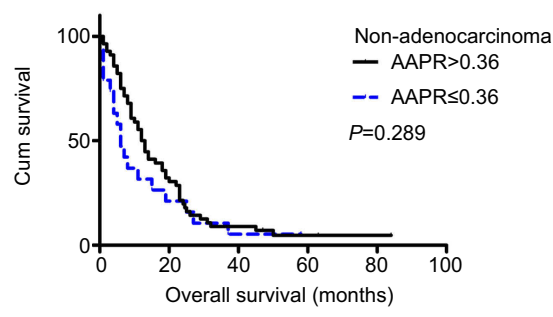

B

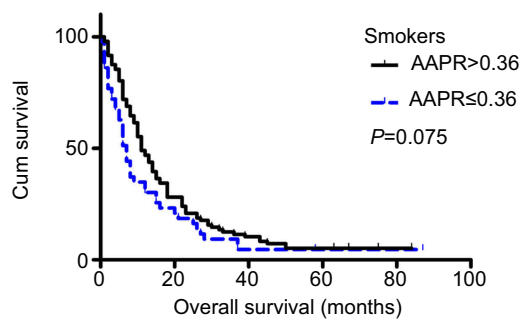

D

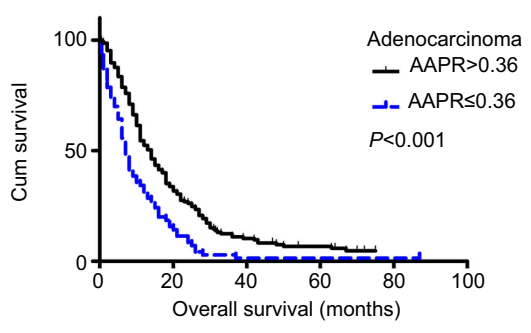

Figure 2 Kaplan-Meier curves of overall survival according to albumin-to-alkaline phosphatase ratio (AAPR) level in (A) all patients, (B) current/past smokers, (C) nonsmokers, (D) adenocarcinoma, and (E) non-adenocarcinoma.

prognosis predictor for metastatic NSCLC patients $P<0.001)$, and LDH level (HR=1.462, 95\% CI $=1.070-1.999$, ( $\mathrm{HR}=0.657,95 \% \mathrm{CI}=0.504-0.856, P<0.05)$. The number of $\quad P<0.05)$ were also significantly related to $\mathrm{OS}$ in multivariate metastatic sites $(\mathrm{HR}=1.418,95 \% \mathrm{CI}=1.026-1.960, P<0.05)$, analysis (Table 3), and their Kaplan-Meier survival curves are systemic therapy $\quad(\mathrm{HR}=0.441, \quad 95 \% \quad \mathrm{CI}=0.338-0.577, \quad$ shown in Figure 3.

Table 3 Prognostic factors for overall survival of metastatic NSCLC patients in univariate and multivariate Cox regression analyses

\begin{tabular}{|c|c|c|c|c|}
\hline \multirow[t]{2}{*}{ Variables } & Univariable & \multirow[b]{2}{*}{$P$-value } & Multivariable & \multirow[b]{2}{*}{$P$-value } \\
\hline & HR $(95 \% \mathrm{CI})$ & & HR $(95 \% \mathrm{CI})$ & \\
\hline Age (years) $(>60 / \leq 60)$ & $1.199(0.945-1.520)$ & 0.143 & $1.107(0.868-1.41 \mathrm{I})$ & 0.413 \\
\hline Gender (female/male) & $1.059(0.83 \mathrm{I}-1.350)$ & 0.643 & & \\
\hline Smoking status (current or past/never) & $1.037(0.819-1.314)$ & 0.763 & & \\
\hline Histology (ADC/non-ADC) & $1.006(0.768-1.317)$ & 0.996 & & \\
\hline Number of metastasis sites $(>2 / \leq 2)$ & $1.362(0.995-1.864)$ & 0.053 & $1.418(1.026-1.960)$ & $0.034 *$ \\
\hline Systemic therapy (yes/no) & $0.426(0.329-0.55 \mathrm{I})$ & $0.000 *$ & $0.44 \mid(0.338-0.577)$ & $0.000 *$ \\
\hline LDH $(>265.5 / \leq 265.5 \mathrm{U} / \mathrm{L})$ & $1.829(1.354-2.470)$ & $0.000 *$ & $1.462(1.070-1.999)$ & $0.017^{*}$ \\
\hline AAPR $(>0.36 / \leq 0.36)$ & $0.587(0.454-0.758)$ & $0.000 *$ & $0.657(0.504-0.856)$ & $0.002 *$ \\
\hline
\end{tabular}

Note: $* P<0.05$.

Abbreviations: AAPR, albumin-to-alkaline phosphatase ratio; ADC, adenocarcinoma; non-ADC, non-adenocarcinoma; LDH, lactate dehydrogenase; NSCLC, non-smallcell lung cancer. 
A

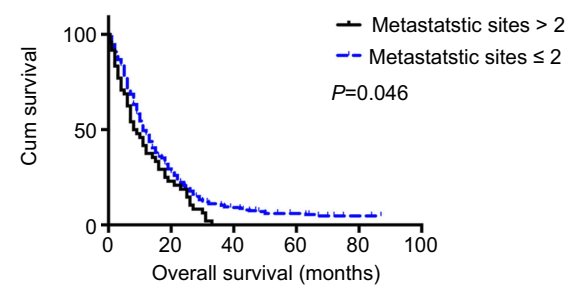

C

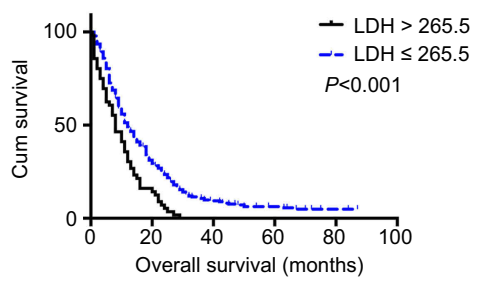

B

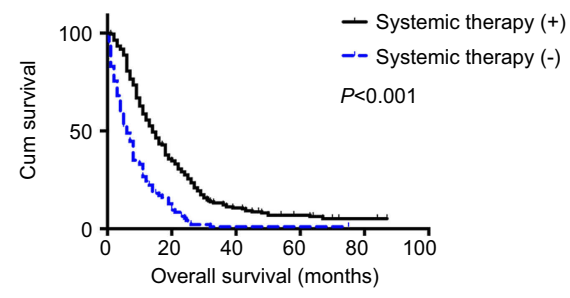

Figure 3 Kaplan-Meier curves of overall survival according to (A) metastatic sites, (B) systemic therapy, and (C) lactate dehydrogenase (LDH).

\section{Discussion}

The main aim of the present retrospective study was to elucidate the associations between the AAPR and survival in patients with metastatic NSCLC. We found that elevated AAPR was correlated with longer survival in metastatic NSCLC patients, especially for patients who were nonsmokers and had confirmed adenocarcinoma. Later, we identified AAPR as an independent favorable prognostic indicator for metastatic NSCLC in multivariate analysis. We also proved that LDH had a prognostic effect in metastatic NSCLC patients.

By multivariate analysis, the high AAPR group was found to be independently related to better survival in metastatic NSCLC. The novel APPR index, calculated from two routine blood parameters, namely ALB and ALP, was first derived in a cohort of hepatocellular carcinoma patients receiving curative surgery, where Chan et al identified that a lower AAPR level increased the risk of early death 3.3-fold and the risk of tumor relapse 2.3 -fold. ${ }^{20}$ A later study retrospectively reviewed 209 metastatic nasopharyngeal carcinoma patients treated with cisplatin-based regimens, and found that higher AAPR indicated longer OS and progression-free survival compared with lower AAPR (24.3 months vs 17.3 months, 8.4 months vs 5.9 months, respectively). ${ }^{21}$ All of these results were consistent with our findings.

We also conducted subanalyses stratified by clinical characteristics, considering that some of the baseline characteristics were uneven between the AAPR $>0.36$ and AAPR $\leq 0.36$ groups. When it comes to subanalyses divided by smoking status and tumor histology types, the discriminatory power of AAPR for OS stayed strong in patients who were non-smokers and had adenocarcinomas, while, in contrast, it became insignificant in those who were smokers and nonadenocarcinoma patients. This result may be explained by the fact that adenocarcinoma was a predominant histological type among lung cancer in non-smokers, while distant metastases such as bone or liver metastases developed earlier and were more prevalent among adenocarcinomas compared to non-adenocarcinomas. ${ }^{25}$ During subanalyses among patients with two or fewer metastasis sites and those with more than two, patients with or without systemic therapy, and those with absent or existing liver/bone metastasis, we found that the tendency remained unchanged, which demonstrates that the AAPR predicted prognosis independently of therapies, number of metastatic sites, and liver/bone metastatic status at diagnosis.

Moreover, in multivariate analysis, we found that having two or fewer metastatic sites, the presence of systemic therapy, and low LDH level were significant related to better survival. Although it is generally acknowledged that the first two conditions predict longer survival than those harboring more than metastatic sites and without systemic therapy, they are not as cheap and convenient as AAPR in predicting prognosis. Regarding LDH, we validated its prognostic value for survival, as indicated in previous studies. ${ }^{10,11}$ AAPR and LDH could supplement each other in clinical application.

There are several shortcomings of our study. First, as this was a retrospective and single-center study, multicenter and well-designed prospective studies are needed to validate our 
findings. Second, although we excluded patients with known liver, renal, and bone diseases to restrict confounding factors, patients with undiscovered liver, renal, or bone diseases could have been mistakenly enrolled in the study. Third, there should be little difference between AAPRs of 0.35 and 0.37 , but they fell on the side of high risk and low risk, respectively, based on the current study, owing to our transforming AAPR into a dichotomous variable when it was actually a continuous one. Moreover, the optimal cut-off value for AAPR requires external validation. Fourth, the mutation status of genes, eg, EGFR, KROS, and EML4$A L K$, and performance status of patients were unknown owing to information loss or unavailable data. Further studies are required to assess correlations between AAPR level and specific gene mutations, and to explore the prognostic impact of the AAPR in NSCLC treated with targeted therapy. Finally, whether dynamic changes in AAPR could be applied to monitor cancer progression and response to therapy in NSCLC remains to be solved in future research.

\section{Conclusion}

AAPR, a simple, inexpensive, and easily gained biomarker-based index, can be an independent predictor for OS in metastatic NSCLC. Further large-scale prospective studies are needed to validate and expand our findings.

\section{Acknowledgments}

This work was supported by the Transformation Projects of Sci-Tech Achievements of Sichuan Province (2016CZYD0001), and the Sci-Tech Support Program of Science and Technology Department of Sichuan Province (2016SZ0073).

\section{Disclosure}

The authors report no conflicts of interest in this work.

\section{References}

1. Siegel RL, Miller KD, Jemal A. Cancer statistics, 2017. CA Cancer J Clin. 2017;67(1):7-30.

2. DeSantis $\mathrm{CE}$, Lin $\mathrm{CC}$, Mariotto $\mathrm{AB}$, et al. Cancer treatment and survivorship statistics, 2014. CA Cancer J Clin. 2014;64(4):252-271.

3. Reck M, Popat S, Reinmuth N, De Ruysscher D, Kerr KM, Peters S. Metastatic non-small-cell lung cancer (NSCLC): ESMO clinical practice guidelines for diagnosis, treatment and follow-up. Ann Oncol. 2014;25 Suppl 3:iii27-39.

4. Ferreira LM, Hebrant A, Dumont JE. Metabolic reprogramming of the tumor. Oncogene. 2012;31(36):3999-4011.
5. Schoppet M, Shanahan CM. Role for alkaline phosphatase as an inducer of vascular calcification in renal failure? Kidney Int. 2008;73(9):989-991. doi:10.1038/ki.2008.104

6. Hung HY, Chen JS, Chien Y, et al. Preoperative alkaline phosphatase elevation was associated with poor survival in colorectal cancer patients. Int J Colorectal Dis. 2017;32(12):1775-1778. doi:10.1007/s00384-0172907-4

7. Namikawa $T$, Ishida $N$, Tsuda $S$, et al. Prognostic significance of serum alkaline phosphatase and lactate dehydrogenase levels in patients with unresectable advanced gastric cancer. Gastric Cancer. 2019 Jul;22(4):684-691. doi:10.1007/s10120-018-0897-8.

8. Kolev Y, Uetake H, Takagi Y, Sugihara K. Lactate dehydrogenase-5 (LDH-5) expression in human gastric cancer: association with hypoxia-inducible factor (HIF-1alpha) pathway, angiogenic factors production and poor prognosis. Ann Surg Oncol. 2008;15(8):2336-2344.

9. Marmorino F, Salvatore L, Barbara C, et al. Serum LDH predicts benefit from bevacizumab beyond progression in metastatic colorectal cancer. Br J Cancer. 2017;116(3):318-323.

10. Zhang X, Guo M, Fan J, et al. Prognostic significance of serum LDH in small cell lung cancer: a systematic review with meta-analysis. Cancer Biomarker. 2016;16(3):415-23.

11. Lee DS, Park KR, Kim SJ, et al. Serum lactate dehydrogenase levels at presentation in stage IV non-small cell lung cancer: predictive value of metastases and relation to survival outcomes. Tumor Biol. 2016;37(1):619625 .

12. McMillan DC, Watson WS, O'Gorman P, Preston T, Scott HR, McArdle CS. Albumin concentrations are primarily determined by the body cell mass and the systemic inflammatory response in cancer patients with weight loss. Nutr Cancer. 2001;39(2):210-213. doi:10.1207/ S15327914nc392_8

13. Deme D, Telekes A. [Prognostic importance of albumin in oncology]. Orv Hetil. 2018;159(3):96-106.

14. Fruchtenicht AV, Poziomyck AK, Kabke GB, et al. Nutritional risk assessment in critically ill cancer patients: systematic review. Rev Bras Ter Intensiva. 2015;27(3):274-283.

15. Fan H, Shao ZY, Xiao YY, et al. Comparison of the Glasgow Prognostic Score (GPS) and the modified Glasgow Prognostic Score (mGPS) in evaluating the prognosis of patients with operable and inoperable non-small cell lung cancer. $J$ Cancer Res Clin Oncol. 2016;142(6):1285-1297. doi:10.1007/s00432-015-2113-0

16. Chang PA-O, Yeh KY, Wang $\mathrm{CH}$, et al. Impact of the pretreatment glasgow prognostic score on treatment tolerance, toxicities, and survival in patients with advanced head and neck cancer undergoing concurrent chemoradiotherapy. Head Neck. 2017;39(10):1990-1996.

17. Shiroyama T, Suzuki H, Tamiya M, et al. Pretreatment advanced lung cancer inflammation index (ALI) for predicting early progression in nivolumab-treated patients with advanced non-small cell lung cancer. Cancer Med. 2018;7(1):13-20.

18. Tokunaga R, Sakamoto Y, Nakagawa S, et al. Prognostic nutritional index predicts severe complications, recurrence, and poor prognosis in patients with colorectal cancer undergoing primary tumor resection. Dis Colon Rectum. 2015;58(11):1048-1057. doi:10.1097/ DCR.0000000000000458

19. Li D, Yuan X, Liu J, Li C, Li W. Prognostic value of prognostic nutritional index in lung cancer: a meta-analysis. $J$ Thorac Dis. 2018;10(9)5298-5307.

20. Chan AW, Chan SL. Albumin-to-alkaline phosphatase ratio: a novel prognostic index for hepatocellular carcinoma. Dis Markers. 2015;2015:1-10.

21. Nie M, Sun P, Chen C, et al. Albumin-to-alkaline phosphatase ratio: a novel prognostic index of overall survival in cisplatin-based chemotherapy-treated patients with metastatic nasopharyngeal carcinoma. J Cancer. 2017;8(5):809-815. doi:10.7150/jca.17536 
22. Beasley MB, Brambilla E, Travis WD. The 2004 World Health Organization classification of lung tumors. Semin Roentgenol. 2005;40 (2):90-97.

23. Vallieres E, Shepherd FA, Crowley J, et al. The IASLC Lung Cancer Staging Project: proposals regarding the relevance of TNM in the pathologic staging of small cell lung cancer in the forthcoming (seventh) edition of the TNM classification for lung cancer. J Thorac Oncol. 2009;4(9):1049-1059. doi:10.1097/JTO.0b013e3181b27799
24. Budczies J, Klauschen F, Sinn BV, et al. Cutoff finder: a comprehensive and straightforward Web application enabling rapid biomarker cutoff optimization. PLoS One. 2012;7(12):e51862. doi:10.1371/journal.pone.0051862

25. Couraud S, Zalcman G, Milleron B, Morin F, Souquet PJ. Lung cancer in never smokers-a review. Eur $J$ Cancer. 2012;48 (9):1299-1311. doi:10.1016/j.ejca.2012.03.007 


\section{Supplementary material}

A

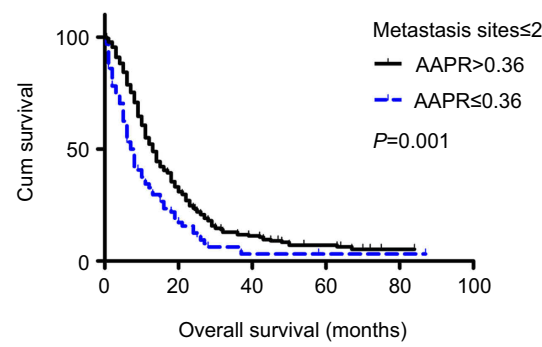

C

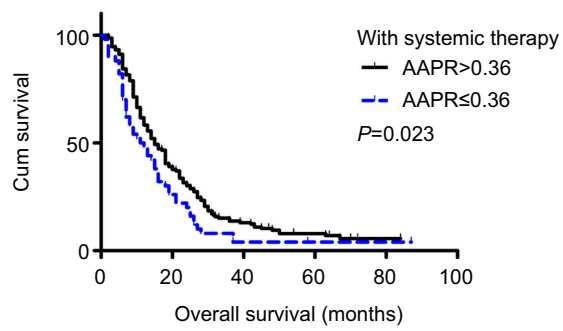

E

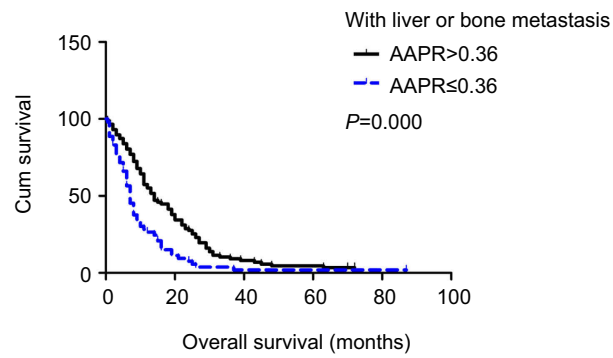

B

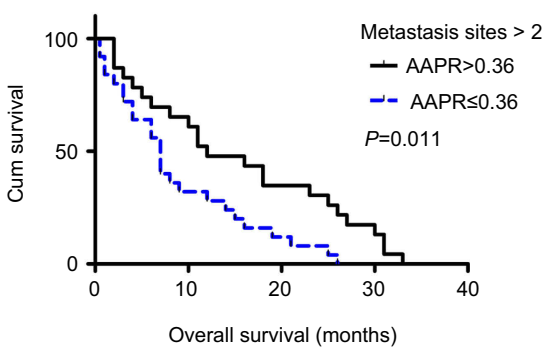

D

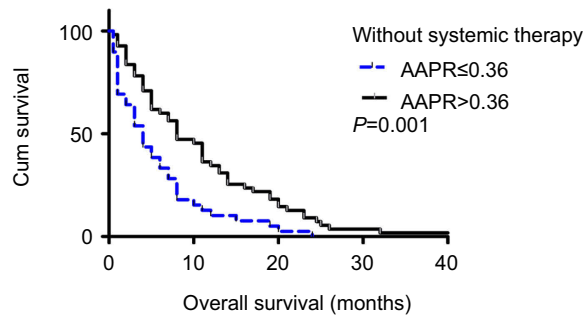

F

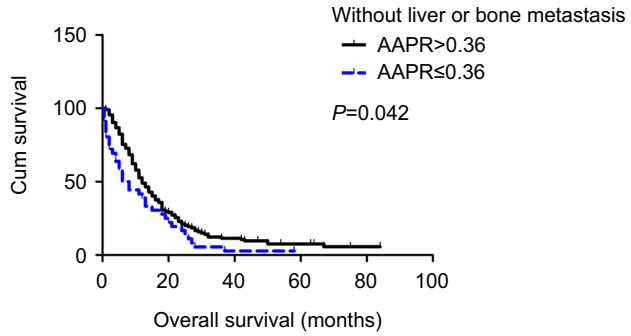

Figure SI Kaplan-Meier curves of overall survival according to albumin-to-alkaline phosphatase ratio (AAPR) level in patients (A) with two or fewer metastasis sites, (B) with more than two metastasis sites, (C) with systemic therapy, (D) without systemic therapy, (E) with liver/bone metastasis, and (F) without liver/bone metastasis.

\section{Publish your work in this journal}

OncoTargets and Therapy is an international, peer-reviewed, open access journal focusing on the pathological basis of all cancers, potential targets for therapy and treatment protocols employed to improve the management of cancer patients. The journal also focuses on the impact of management programs and new therapeutic agents and protocols on patient perspectives such as quality of life, adherence and satisfaction. The manuscript management system is completely online and includes a very quick and fair peer-review system, which is all easy to use. Visit http://www.dovepress.com/ testimonials.php to read real quotes from published authors. 